\title{
THE CLAMP FORCE CONTROL OF TRAIN ELECTRO-MECHANICAL BRAKE DEVICE ON SELF-ADAPTATION CONTROL
}

\author{
LEI CHI \& WU MENG-LING \\ Institute of Railway and Urban Mass Transit, Tongji University, China
}

\begin{abstract}
The train electro-mechanical brake (EMB) device, which is different from pneumatic brake and hydraulic brake, is a new type of brake mode. The train EMB device consists of motor-servo system and mechanical transmission. Due to nonlinear friction of the motor and uncertain resistance of transmission, the clamp force can't be controlled accurately with open loop. In this paper, the nonlinear friction of the motor and the uncertain resistance of transmission are regarded as disturbing force. A self-adaptation estimator which can estimate the disturbing force online is designed. The input of train EMB device, which is compensated by the output of estimator, makes controller adapted timevarying disturbing. To verify the clamp force control performance, a Hardware-In-the-Loop (HIL) test is carried out. Test results suggest that the proposed self-adaptation controller is able to provide valid estimated disturbing force and control the clamp force accurately.

Keywords: electro-mechanical brake (EMB), clamp force, disturbing force, self-adaptation estimator, Hardware-In-the-Loop (HIL) test.
\end{abstract}

\section{INTRODUCTION}

The key question of the train Electro-mechanical brake (EMB) device is how to control the clamp force accurately. For EMB device, nonlinear friction of motor and uncertain resistance of transmission are two important factors restricting its precise control of clamp force.

On the one hand, friction of motor is commonly simulated by friction model at present. Static friction, Coulomb friction and viscous friction model are common models which can simulate friction of motor to a certain extent as well. However, their consideration about nonlinear friction is still insufficient [1]. Stribeck friction model has considered the interference from nonlinear friction of motor, but the empirical parameters of it require a large number of motor performance tests, which seems to limit its application. On the other hand, for resistance of transmission, at present, most engineers have obtained transmission error by combining principle analysis and real-time measurement according to different mechanical transmission modes, which puts forward more stringent requirements for system hardware and measurement precision [3] - [6].

This paper firstly illuminates the system composition of EMB device, as well as the further analysis of disturbing. Then, the paper designs a self-adaptation estimator which can estimate the disturbing force online according to the electric control theory, and relevant system identification has been completed as well. Finally, the control performance of the algorithm is verified by the HIL test.

\section{SYSTEM STRUCTURE AND DISTURBING ANALYSIS}

\subsection{System structure}

The train EMB system consists of motor-servo system, mechanical transmission and sensor. Its system block diagram is shown in the Fig. 1. The motor adopts the current loop vector control, and the armature current will follow the target current, which can be expressed as a 


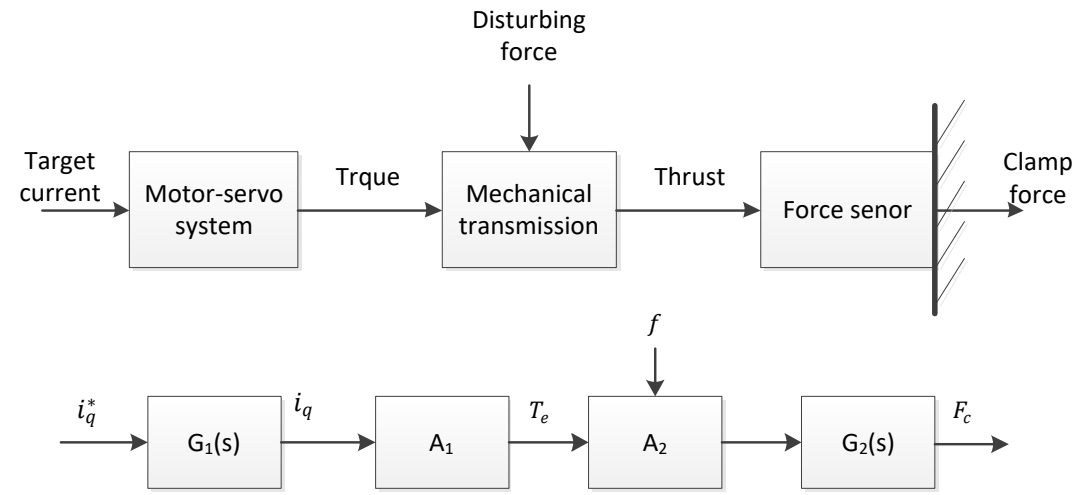

$i_{q}^{*}$ - target current; $i_{q}$ - armature current; $T_{e}$ - torque; $F_{c}$ - clamp force; $f$ - disturbing force

Figure 1: System block diagram of EMB.

transfer function $G_{1}(s)$ with a gain of 1 . When the motor works, the torque is generated proportionally according to the armature current, and the ratio is $A_{1}$. The mechanical transmission device can convert the torque to the thrust at a certain rotating speed ratio, which is $A_{2}$. Finally, the clamp force is produced by compression deformation of the friction pair, which is essentially a process of rapid rise or rapid decline, which can be expressed by $G_{2}(s)$.

\subsection{Disturbing analysis}

Due to machining accuracy, assembly accuracy and some inherent properties, motor and mechanical transmission have certain error and resistance when they produce and transmit force, and the error and resistance are time-varying and nonlinear. In this paper, the nonlinear friction of motor and the uncertain resistance of transmission are regarded as a disturbing force. And the disturbing force ( $f$ in system block diagram of EMB) acting on transmission links is an unknown value.

To further illustrate the time-varying characteristics and nonlinearity of the disturbing force and identify the intrinsic gains $\mathrm{A}_{1}$ and $\mathrm{A}_{2}$ in the system, the open-loop test of EMB device is carried out, which is shown in Fig. 2.

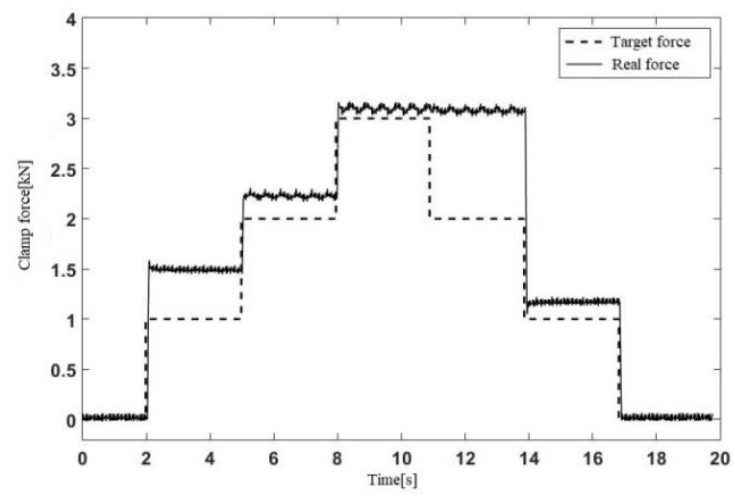

Figure 2: Open-loop step response of EMB. 
According to the open-loop test, the ideal value of $A_{1}$ and $A_{2}$ are 0.92 and 1.2 respectively. then the ideal value of $\mathrm{A}_{1} \cdot \mathrm{A}_{2}$ is 1.104 . The disturbing force $f$ equals the difference between the product of input value times $\mathrm{A}_{1} \cdot \mathrm{A}_{2}$ and output of system. As can be seen from Fig. $2, f$ is not the same when the clamp force is rising and declining, which causes the system cannot be precisely controlled.

\section{CONTROLLER DESIGN}

\subsection{Estimation algorithm}

As can be seen from the last section, the main reason that the clamp force of the system is difficult to control precisely is the model uncertainty caused by mechanical interference. In this paper, a self-adaptation control algorithm is designed to control the clamp force of the EMB device. This algorithm adapts to nonlinear time-varying system, of which control principle is, firstly, by using the control quantity and the controlled object to design a selfadaptation estimator to estimate the disturbing force $f$ and then using the feedforward compensation to obtain the precise control of the clamp force. The structure of control is shown in Fig. 3.

As shown in Fig. $3, \hat{f}$ is the estimation of disturbing force $f$.

The relationship between output $F_{c}$ and input $i_{q}^{*}$ of the EMB device is as follows:

$$
F_{c}(k)=A_{1} A_{2} G_{1}(s) G_{2}(s) i_{q}^{*}(k)-G_{2}(s) f(k)
$$

In eqn (1), $k$ represents at the moment of $k$.

The algorithm for estimating the parameters of disturbing force $f$ is as follows:

$$
\hat{f}(k)=\frac{1}{k} \sum_{i=1}^{k}\left(A_{1} A_{2} \hat{G}(s) i_{q}^{*}(k)-F_{c}(k)\right)
$$

In eqn (2) above, $\widehat{G}(s)$ is the estimation of $G_{1}(s) G_{2}(s)$ in the controlled object.

Converting to a recursive formula is as follows:

$$
\hat{f}(k+1)=\frac{1}{k+1}\left(k \hat{f}(k)+A_{1} A_{2} \hat{G}(s) i_{q}^{*}(k)-F_{c}(k)\right)
$$

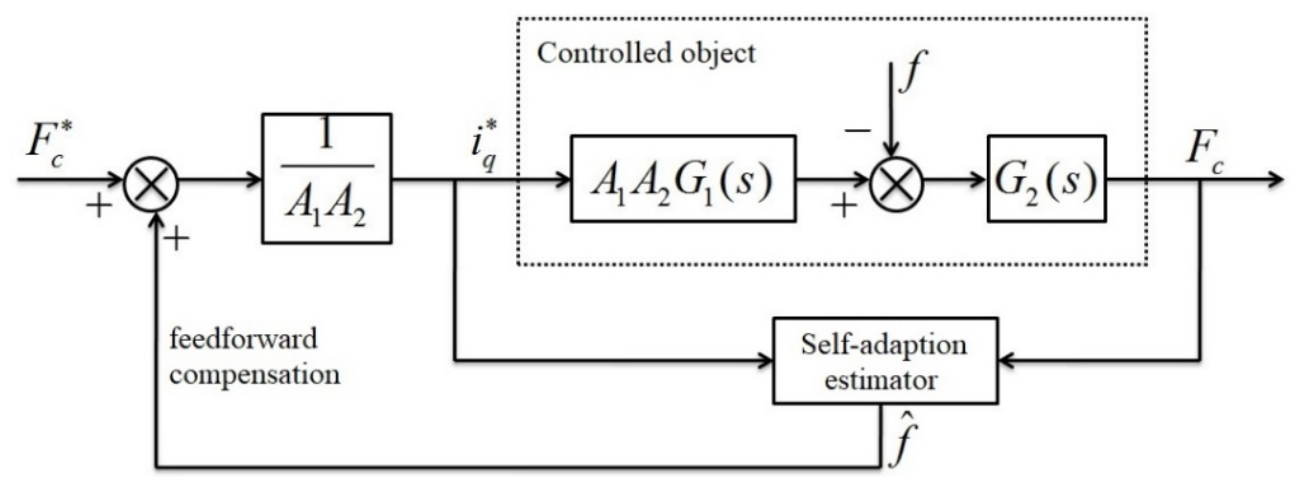

Figure 3: Structure of control. 
From a statistic point of view, the estimation of disturbing force $f$ by eqns (1)-(3) is a kind of arithmetical averaging method, that is, to average the deviation of historical data $\mathrm{A}_{1} \mathrm{~A}_{2} \widehat{\mathrm{G}}(\mathrm{s}) \mathrm{i}_{\mathrm{q}}^{*}(\mathrm{k})$ and system output $F_{c}(k)$. However, for this system, because of all kinds of interference, the calculated value of disturbing force is time-varying, the algorithm should emphasize the effect on the latest data, and the old data should be gradually forgotten. The forgetting factor $\mathrm{b}$ can be introduced, that is, the weighted coefficient of each historical data is given over time, and the recursive method is as follows:

$$
\begin{gathered}
d_{k}=\frac{1-b}{1-b^{k+1}} \\
\hat{f}(k+1)=\left(1-d_{k}\right) \hat{f}(k)+d_{k}\left(A_{1} A_{2} \hat{G}(s) i_{q}^{*}(k)-F_{c}(k)\right)
\end{gathered}
$$

Then the estimated $\hat{f}$ is compensated for the system output as feedforward, which can make the actual output of system to converge with the target output.

\subsection{System identification}

To illustrate the influence of accuracy of $\widehat{\mathrm{G}}(\mathrm{s})$ on the control performance of self-adaptation control algorithm, Simulink simulation platform is used to simulate the self-adaptation control performance in two cases that $\widehat{\mathrm{G}}(\mathrm{s})$ is accurate and inaccurate. In the simulation, suppose that $G_{1}(s) G_{2}(s)$ in the controlled object is first order damp elements $\frac{1}{0.05 s+1}$, disturbing force $f$ is $0.5 \mathrm{kN}$, forgetting factor $\mathrm{b}$ is 0.9 , and the target clamp force is set at $1 \mathrm{kN}$. When $\widehat{G}(s)$ are taken 1 (simulation 1) and $\frac{1}{0.05 \mathrm{~s}+1}$ (simulation 2), the control performance is shown in Fig. 4.

Compared simulation 1 with simulation 2 , it can be found that control of clamp force in simulation 1 is oscillating, and its control performance is far less than that in simulation 2. This result shows that the accuracy of $\widehat{\mathrm{G}}(\mathrm{s})$ has a great impact on the control performance of

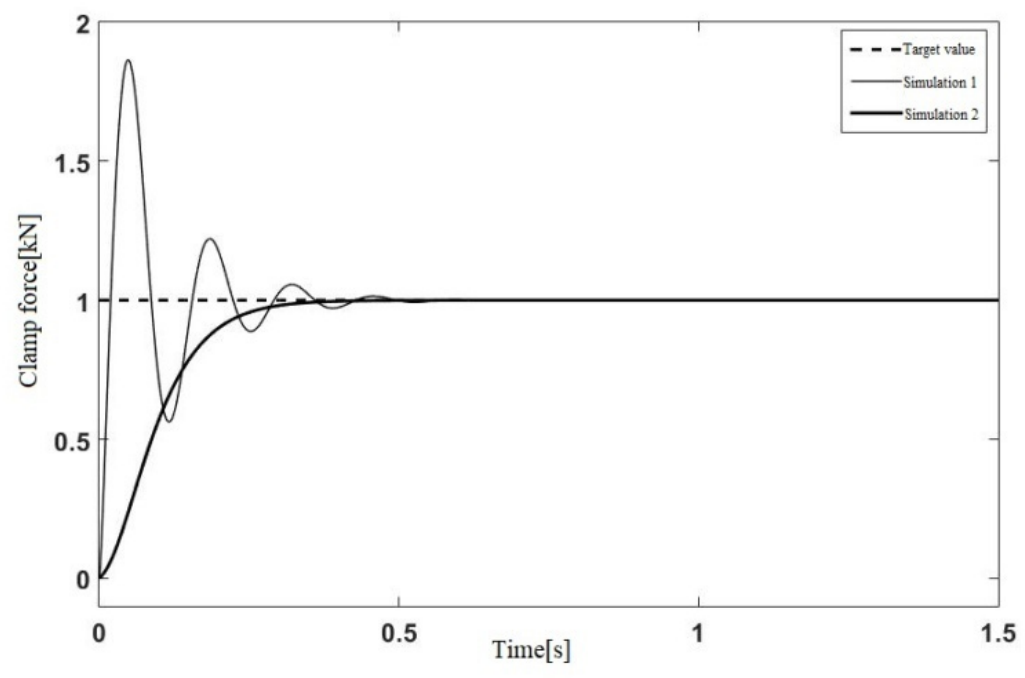

Figure 4: Comparing of control performance. 
self-adaptation controller, the closer $\widehat{G}(\mathrm{~s})$ is to $\mathrm{G}(\mathrm{s})$, the better the control performance is. Therefore, it is necessary to make simple system identification to $\widehat{G}(s)$ in the controlled object.

To facilitate the adjustment of parameters, the following two single parameter dynamic systems are selected as reference models.

First order damp elements:

$$
x(k+1)=x(k)+h \cdot \lambda \cdot(x(k)-u(k))
$$

Discrete fastest feedback system:

$$
\left\{\begin{array}{l}
f h=\operatorname{fhan}\left(x_{1}(k)-u(k), x_{2}(k), r, h\right) \\
x_{1}(k+1)=x_{1}(k)+h \cdot x_{2}(k) \\
x_{2}(k+1)=x_{2}(k)+h \cdot f h
\end{array}\right.
$$

In eqns (6) and (7), $h$ is compute cycles, $\lambda$ is transition frequency of first order damp elements, $r$ is tracking acceleration of discrete fastest feedback system, $\operatorname{fhan}\left(x_{1}, x_{2}, r, h\right)$ is the fastest synthesis function [7]

Fig. 5 show the step response curves of first order damp element (reference model 1), discrete fastest feedback system (reference model 2) and the real force of the EMB device.

In Fig. 5, comparing the recombination of step response curves of the two reference models and plant, the discrete fastest feedback system is closer to the clamp force step response of the EMB device, and the identification value of $r$ is 1000 . Then $\widehat{\mathrm{G}}(\mathrm{s})$ is selected as the discrete fastest feedback system with the tracking acceleration of 1000 .

Through further experiments, identifying the key parameters in the system model and algorithm, finally, the forgetting factor $b$ is 0.99 , the fixed gain of the controller $\frac{1}{A_{1} A_{2}}$ is determined as $\frac{1}{1.5}$, and the control dead band is set at $0.05 \mathrm{kN}$, to avoid excessive control over the loss of hardware.

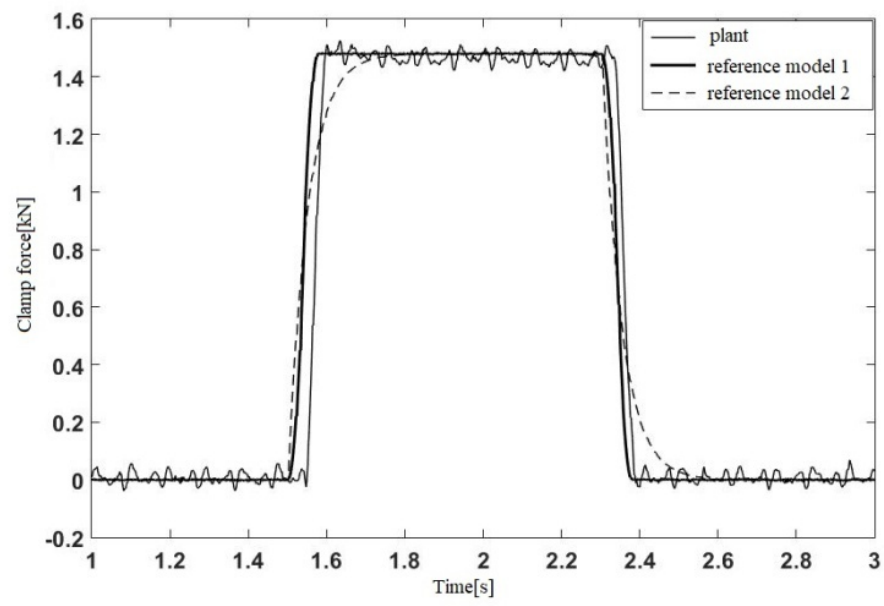

Figure 5: System identification performance. 


\section{EXPERIMENTAL RESULTS}

\subsection{Experimental setup}

HIL test will be carried out to test the self-adaptation algorithm of clamp force presented in Section 3. HIL test environment is shown in the Fig. 6. This test device includes two parts: simulation software and hardware. Among them, the simulation software is carried on the host computer, including the logic operation module, the collection filter module and the instruction issue module. The simulation software is mainly used for the realization of the self-adaptation algorithm, the clamp force data acquisition, data processing and the issuing of control instructions. The hardware includes the EMB device (1, 2, 3, 4 in Fig. 6), motor driver, host computer and power. The process of HIL test is as follows :

1. The control instructions generated by the host computer are transmitted to motor driver over CAN network;

2. Motor driver uses the current loop vector to drive the motor according to the control instructions;

3. Motor converts the motion through mechanical transmission, resulting in clamp force;

4. Force sensor collects the clamp force, and sends the signal to the host computer;

5. According to the signal of clamp force, the host computer estimates the disturbing force, realizes the self-adaptation algorithm, and completes the close-loop control.

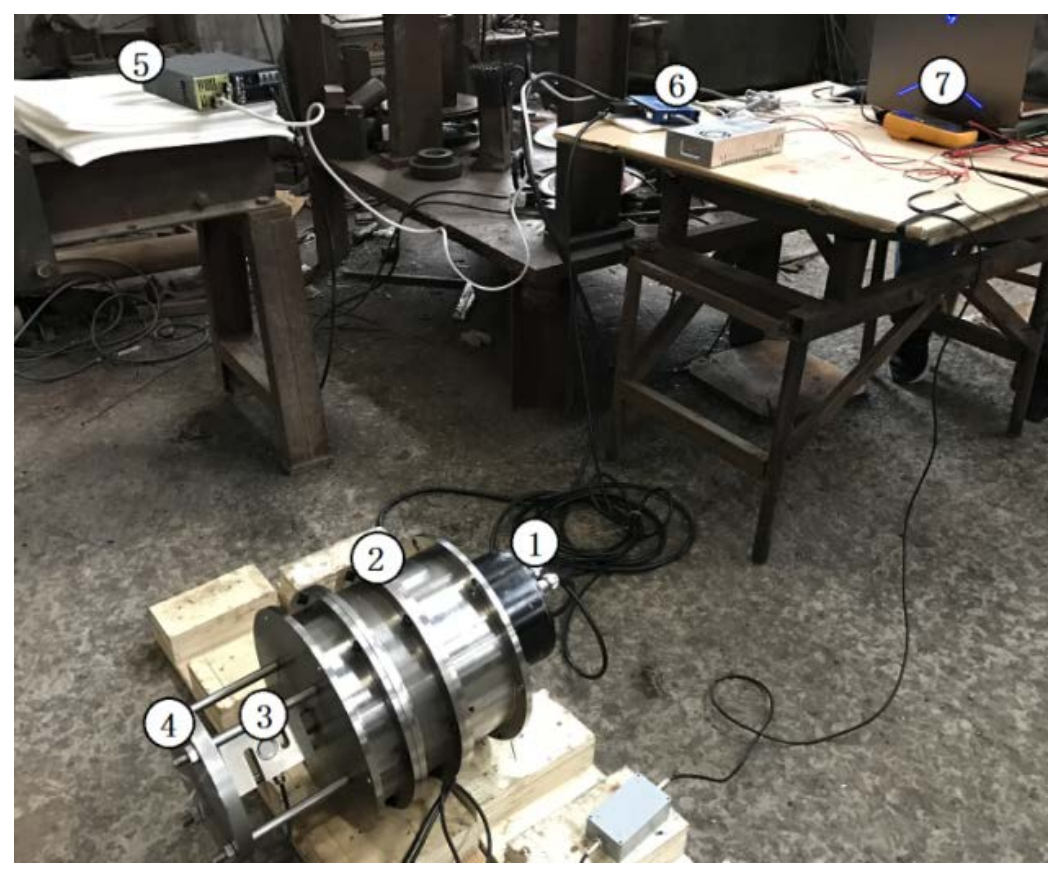

1 - Motor; 2 - Mechanical transmission; 3 - Force sensor;

4 - Friction pair; 5 - Power; 6 - Motor driver; 7 - Host computer

Figure 6: HIL test environment. 


\subsection{Results}

In this section, the control performance of the clamp force self-adaptation algorithm proposed in this paper is verified by the HIL test. Two kinds of simulation experiments are carried out: step response test; rise and decline by stages test.

\subsubsection{Step response test}

The value of target force in the test is $2 \mathrm{kN}$, and its clamp force step response is shown in Fig. 7.

In Fig. 7, the response time $t_{r}$ of clamp force in the rising process is $0.14 \mathrm{~s}$, and overshoot $M_{p}$ is $0.1 \mathrm{kN}$; the response time of clamp force in the declining process is $0.12 \mathrm{~s}$, and overshoot is $0.02 \mathrm{~N}$. Real force can quickly reach the requirement of target force, meanwhile maintain a stable value.

Estimated value of disturbing force $\widehat{f}$ based on step response test is shown in Fig. 8.

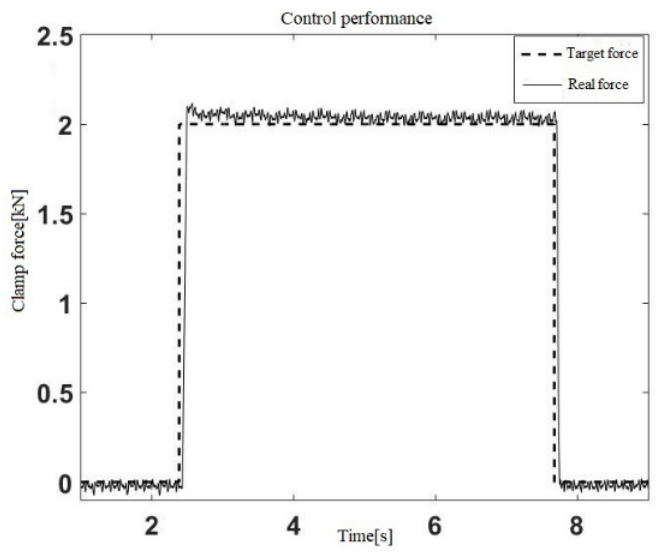

Figure 7: Step response curve.

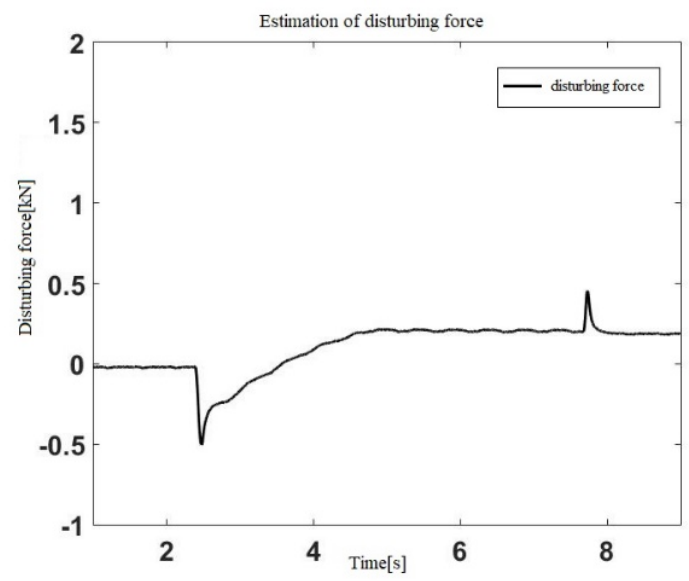

Figure 8: $\hat{f}$ of step response. 
As can be seen from Fig. 8, in the moment that clamp force is established and eliminated, the EMB device has a mutational disturbing force, which is actually the uncertain force generated during start-stop process of the motor and transmission. The self-adaptation algorithm successfully quantifies this phenomenon. While in the process of holding clamp force, the disturbing force fluctuates slightly in a relatively stable range.

\subsubsection{Rising and declining by stages test}

In order to comprehensively test the control performance under various clamp force values, in the test process the target force is set as a mode, which is rising and declining by stages. Target force for each stage is $0 \rightarrow 1 \rightarrow 2 \rightarrow 3 \rightarrow 2 \rightarrow 1 \rightarrow 0 \mathrm{kN}$, and its clamp force response is shown in Fig. 9.

The response time and overshoot of clamp force step response calculated in each stage is shown in Table 1.

As can be seen from Fig. 9 and Table 1, the maximum overshoot $M_{p \max 1}$ of clamp force at rising stage is $0.15 \mathrm{kN}$, and this value exists in the process of $0 \rightarrow 1 \mathrm{kN}$; The maximum overshoot $M_{p \max 2}$ of clamp force at declining stage is $0.1 \mathrm{kN}$, and this value exists in the process of $2 \rightarrow 1 \mathrm{kN}$. Therefore, real force can follow target force well.

Estimated value of disturbing force $\hat{f}$ based on rising and declining by stages test is shown in Fig. 10.

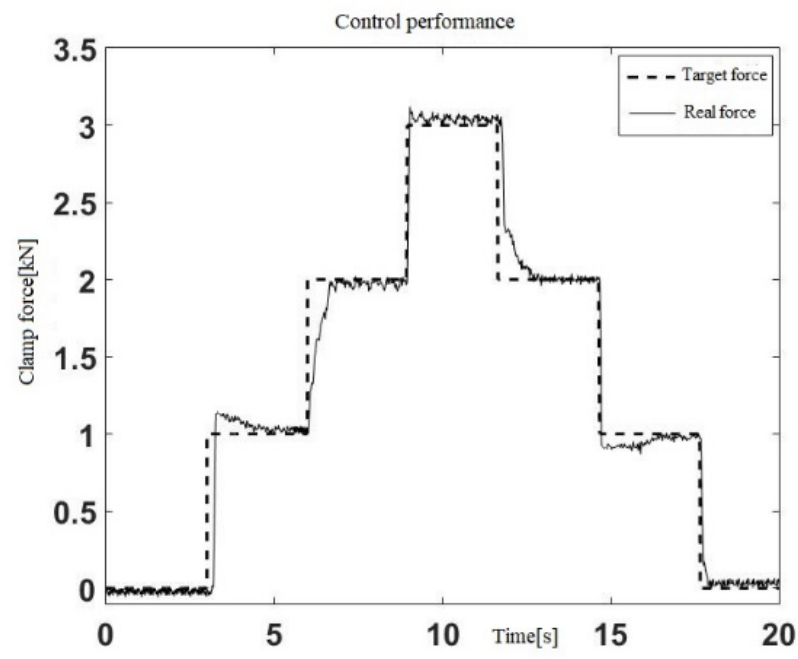

Figure 9: Rising and declining by stages curve and $\hat{f}$.

Table 1: Response time and overshoot.

\begin{tabular}{|c|c|c|c|c|c|c|}
\hline \multirow{2}{*}{$\begin{array}{c}\text { Target force } \\
(\mathrm{kN})\end{array}$} & \multicolumn{3}{|c|}{ Rising stage } & \multicolumn{3}{c|}{ Declining stage } \\
\cline { 2 - 7 } & 1 & 2 & 3 & 2 & 1 & 0 \\
\hline$t_{r}(\mathrm{~s})$ & 0.17 & 0.8 & 0.09 & 1.11 & 0.09 & 0.1 \\
\hline$M_{p}(\mathrm{kN})$ & 0.15 & 0 & 0.05 & 0 & 0.1 & 0 \\
\hline
\end{tabular}




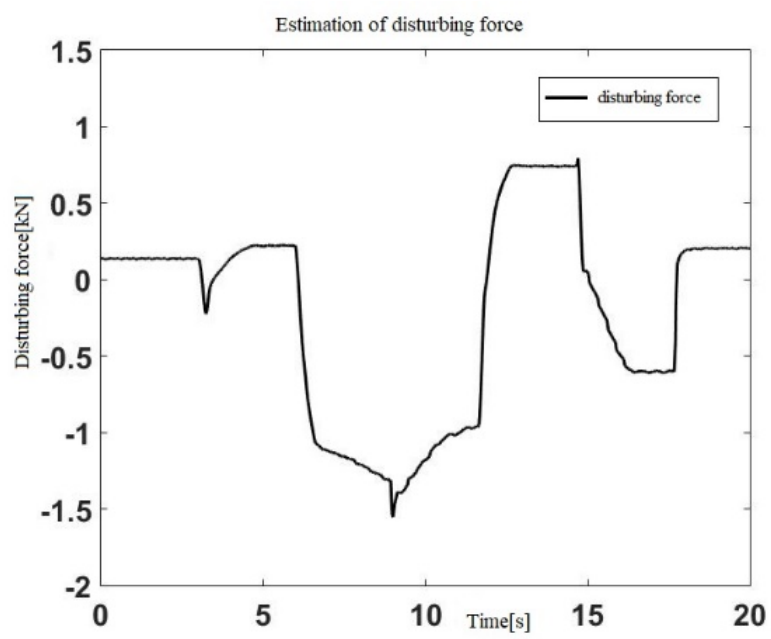

Figure 10: $\hat{f}$ of rising and declining by stages.

As can be seen from Fig. 10, the self-adaptation algorithm can still effectively predict the mutational disturbing force of the EMB device at the moment of change in the clamp force stage.

Compared Fig. 9 with Fig. 2, under the same working condition, the maximum overshoot of open-loop control is about $0.5 \mathrm{kN}$, while the maximum overshoot of the control method of self-adaptation algorithm proposed in this paper is only $0.15 \mathrm{kN}$. Therefore, the selfadaptation algorithm proposed in this paper can estimate disturbing force well. Furthermore, the clamp force can be controlled rapidly and precisely under the premise of minimal overshoot.

\section{CONCLUSION}

This paper analyzes firstly factors that cause the output error of the train EMB device, points out that clamp force cannot be controlled under open loop, and then regards nonlinear friction of motor and uncertain resistance of transmissions as disturbing force, uses a self-adaptation estimator to estimate its value and compensates to input as feedforward. Finally, HIL test is used to verify that the algorithm proposed in this paper is feasible. Conclusions are as follows:

- As the EMB device has friction of motor and resistance of transmission, clamp force cannot by controlled in the open loop state.

- The model of EMB device is identified by the discrete fastest feedback system, and a more precise model is obtained, and the control performance of the algorithm is improved.

- Under the control algorithm of this paper, the disturbing force can be estimated well, and the clamp force is adaptive.

\section{REFERENCES}

[1] Line, C., Manzie, C. \& Good, M.C., Electromechanical brake modelling and control: from PI to MPC. Transactions on Control Systems Technology, 16(3), 2008. 
[2] Line, C., Manzie, C. \& Good, M.C., Control of an electromechanical brake for automotive brake-by-wire systems with an adapted motion control architecture. SAE Automotive Dynamics, Stability \& Controls Conference and Exhibition, 2004.

[3] Youliang, L., Design and Implementation of Control System of Test Analysis and Running-In of Harmonic Gear Reducer, Harbin Institute of Technology, 2014.

[4] Xianqing, L. \& Zhenhua, C., Analyses of transmission error of mechanical shift gear in HMCVT. China Mechanical Engineering, 26(23), pp. 3253-3256, 2015.

[5] Liping, T., Error compensation and fault diagnosis of ball screw and nut. China Science and Technology Information, 23, pp. 73-76, 2006.

[6] Zeyin, H., Transmission Error Analysis and Optimization Research on Vibration and Noise of Gear System, Chongqing University, 2015.

[7] Jingqing, H., Active disturbance rejection control technique. Frontier Science, 25(2), pp. 98-102, 2003. 